\title{
Right ventricular free wall longitudinal strain as determined by speckle tracking echocardiography is a noninvasive predictor of acute cellular rejection in adult heart transplantation
}

Cecília Beatriz Bittencourt Viana Cruz ( $\nabla$ cbbvc@yahoo.com.br)

Universidade de Sao Paulo Instituto do Coracao https://orcid.org/0000-0002-2932-766X

Ludhmila A. Hajjar

Universidade de Sao Paulo Instituto do Coracao

Fernando Bacal

Universidade de Sao Paulo Instituto do Coracao

Marco S. Lofrano-Alves

Universidade Federal do Parana Hospital de Clinicas

Márcio S.M. Lima

Universidade de Sao Paulo Instituto do Coracao

Maria C. Abduch

Universidade de Sao Paulo Instituto do Coracao

Marcelo L.C. Viera

Universidade de Sao Paulo Instituto do Coracao

Hsu P. Chiang

Universidade de Sao Paulo Instituto do Coracao

Juliana B. C. Salviano

Universidade de Sao Paulo Instituto do Coracao

isabela Bispo Santos da Silva Costa

Universidade de Sao Paulo Instituto do Cancer do Estado de Sao Paulo

Julia Tizue Fukushima

Universidade de Sao Paulo Instituto do Cancer do Estado de Sao Paulo

Joao C.N. Sbano

Universidade de Sao Paulo Instituto do Coracao

Wilson Mathias

Universidade de Sao Paulo Instituto do Coracao

Jeane M. Tsutsui

Universidade de Sao Paulo Instituto do Coracao 
Research article

Keywords: heart transplantation, acute cellular rejection, speckle tracking echocardiography

Posted Date: June 24th, 2020

DOI: https://doi.org/10.21203/rs.3.rs-36813/v1

License: (c) (i) This work is licensed under a Creative Commons Attribution 4.0 International License. Read Full License 


\section{Abstract \\ Background}

Acute cellular rejection (ACR) is a major complication after heart transplantation. Endomyocardial biopsy (EMB) remains the gold standard for its diagnosis, but with concerning complications. We evaluated the usefulness of speckle tracking echocardiography (STE) and the biomarkers for detecting ACR after heart transplantation.

\section{Methods}

We prospectively studied 60 transplant patients with normal left and right ventricular systolic function who underwent EMB for surveillance sixth months after transplantation. Sixty age- and sex-matched healthy individuals constituted the control group. Conventional echocardiographic parameters left ventricular global longitudinal, radial and circumferential strain (LV-GLS, LV-GRS and LV-GCS, respectively), left ventricular systolic twist (LV-twist) and right ventricular free wall longitudinal strain (RVFWLS) were analyzed just before procedure. We also measured biomarkers at the same moment.

\section{Results}

Of 60 patients included, $17(28 \%)$ had severe ACR (grade $\geq 2 R$ ) and $43(72 \%)$ had no significant ACR (grade $0-1 \mathrm{R}$ ). Absolute values of LV-GLS, LV-twist and RV-FWLS were lower in transplant patients with ACR degree $\geq 2 \mathrm{R}$ compared with those without ACR $\left(12.5 \% \pm 2.9\right.$ vs $14.8 \% \pm 2.3, p=0.002 ; 13.9^{\circ} \pm 4.8$ vs $17.1^{\circ} \pm 3.2, p=0.048 ; 21.4 \% \pm 3.2$ vs $16.6 \% \pm 2.9, p<0.001$; respectively), while no differences were observed in both LV-GRS or LV-GCS. All these parameters were lower in transplant group without ACR than in non-transplant controls, except for LV-twist. Cardiac troponin I levels were significantly higher in patients with significant ACR than in patients without significant ACR [0.19 (0.09-1.31) vs. 0.05 (0.010.18), $p=0.007$ ]. In multivariate analysis, RV-FWLS was the only independent predictor of ACR (odds ratio $=0.57 ; 95 \% \mathrm{Cl}=0.40-0.81 ; p=0.02)$. RV-FWLS $<18.0 \%$ had an accuracy of $85 \%$ to predict ACR.

\section{Conclusion}

Heart transplant patients have altered left ventricular dynamics compared with non-transplant individuals. RV-FWLS was the only independent predictor of ACR after heart transplantation and should be considered a valuable noninvasive screening parameter for its detection.

\section{Introduction}

Heart transplantation is the choice treatment for select patients with end-stage heart failure $(1,2)$. Although significant advances in immunosuppressive therapy have been beneficial in decreasing cardiac 
allograft rejection, graft failure remains one of the major associated complications $(3,4)$. For this reason, adequate monitoring of heart transplant patients to diagnose and initiate specific therapy for transplant rejection in a timely manner is important, albeit challenging.

Endomyocardial biopsy (EMB) is the widely accepted gold standard for the diagnosis of acute cellular rejection (ACR) (5). However, it is invasive and is associated with complications in 0.2 to $5.5 \%$ of cases; these complications include tricuspid regurgitation, cardiac perforation and cardiac tamponade (6-9). Additional limitations of EMB include subjectivity of the pathological analysis and also significant interobserver variability, which compromises reliability and reproducibility (10-12).

Therefore, there has been much effort to develop noninvasive and accurate methods that can reduce the need for $\mathrm{EMB}$, including biomarker detection, imaging techniques and genetic tests $(13,14)$. The most used biomarkers in patients with heart transplantation are troponin and BNP, however there is a considerable heterogeneity among studies about the time dosage and the predict value for detect ACR $(15,16)$.

Speckle tracking echocardiography (STE) is a newly incorporated technique in clinical practice based on identification and frame-by-frame tracking of natural acoustic myocardial markers that arise from the interaction of ultrasound waves and small myocardial elements. These speckle patterns are unique in each segment, and changes in their positions enable the determination of multiple parameters involved in cardiac mechanics.

In the present study, we aimed to assess the value of STE derived strain measurements and biomarkers for the noninvasive detection of ACR after heart transplantation. We also evaluated the ventricular dynamics of heart transplant patients in comparison with control individuals using STE.

\section{Methods}

\section{Patients}

From January 2014 to November 2018, we prospectively studied heart transplant patients who underwent EMB in the $6^{\text {th }}$ month after orthotopic heart transplantation for the diagnosis of ACR for surveillance. All the included patients were asymptomatic and did not present any hemodynamic compromise at the time of enrollment.

The exclusion criteria were as follows: age $<18$ years, arrhythmia, left ventricular ejection fraction $<0.55$, right ventricular dysfunction of any degree (FAC $<35 \%$ ), vascular graft disease, humoral rejection, two or more previous cellular rejection episodes, chronic kidney disease, Chagas disease reactivation, limited echocardiographic window for STE analysis and inconclusive EMB analysis.

All patients underwent the same protocol according to predefined steps. First, enrolled patients underwent complete echocardiographic analysis for assessment of left and right ventricular function. For those with 
normal systolic function, images were acquired for mechanical analysis by STE, and blood was withdrawn for biomarker tests. The patients then underwent EMB and, according to the results, were divided into the following groups: 1 ) without significant ACR (grades 0 and $1 R$ ) and 2) with significant ACR (grades 2R and 3R).

Individuals without a clinical history of any disease known to interfere with myocardial physiology or structure and who were matched by sex and age with the transplant patients constituted the control group. This group underwent STE for analysis of ventricular mechanics and was compared with the groups of transplant patients with and without significant ACR. The study protocol was approved by our ethical committee, and all patients gave written informed consent to participate.

\section{Endomyocardial biopsy}

EMB was performed through the internal jugular or femoral vein under radioscopy. A minimum of 3 distinct ventricular myocardium fragments were collected (each consisting of at least $50 \%$ myocardium) and sent for anatomopathological analysis. A sample was considered sufficient when at least 3 myocardial fragments were obtained for analysis by optical microscopy after fixation in $10 \%$ formalin and staining of the laminae with hematoxylin and eosin. Two experienced cardiac pathologists blinded to the echocardiographic results analyzed all biopsies. The grade of rejection was based upon the recommendations of the International Society for Heart and Lung Transplantation (17). The results of EMB were described as grade 0 (without rejection), grade $1 \mathrm{R}$ (mild rejection, low grade), grade $2 \mathrm{R}$ (moderate rejection, intermediate grade) or grade 3R (severe rejection, high grade) (17). In our study, grades $2 R$ and $3 R$ were considered to indicate significant $A C R$.

\section{Echocardiography}

On the day of EMB, just before the procedure, patients underwent echocardiographic examination on a commercially available machine equipped with an MS5 probe (GE Vivid 9, GE Healthcare, Milwaukee, Wisconsin, USA). Image acquisition and assessment were performed according to the recommendations of the American Society of Echocardiography (18). Left ventricular ejection fraction was obtained by Simpson's rule throughout apical 4- and 2-chamber views, and left ventricular mass was calculated using the equation proposed by Devereux et al. (19), indexed by body surface area to derive the left ventricular mass index. Right ventricular parameters were analyzed in the apical 4-chamber view focused on the right ventricle. Right ventricular systolic function was assessed with the conventional parameters recommended for routine clinical practice: tricuspid annular plane systolic excursion, systolic excursion velocity, and fractional area change, which were obtained with M-mode, pulsed tissue Doppler and twodimensional echocardiography, respectively. Diastolic function was evaluated based on mitral inflow E/A pattern, E/A ratio, E velocity deceleration time, annular tissue Doppler curves (e'/a'), and E/e' ratio.

To assess cardiac mechanics, 3 consecutive cardiac cycles were recorded. Left ventricular short-axis and apical views were acquired using two-dimensional grayscale second-harmonic imaging at a frame rate of 50-80 frames per second. Left ventricular short-axis views at the basal, mid (papillary muscles) and apex 
levels were acquired to analyze circumferential and radial strain, while left ventricular apical 4-, 2- and 3chamber views were used to assess left ventricular global longitudinal strain (LV-GLS) and an apical 4chamber view was used to analyze right ventricular free wall longitudinal strain (RV-FWLS).

Speckle tracking echocardiography analysis was performed offline using dedicated software (EchoPAC, version BT11, GE Healthcare). All echocardiographic measurements were performed by one specialist blinded to the clinical data. End-systole was determined by pulsed-wave Doppler at the time of aortic valve closure. After the ventricular endocardial border was manually traced, the epicardial borders were automatically defined to create regions of interest according to the ventricular segmentation; if necessary, adjustments were made by the operator. In particular, care was taken not to include the myocardial trabeculae and the pericardium. Following this step, myocardial speckles were automatically tracked by dedicated software and, in the case of suboptimal tracking, further manual adjustments were allowed, resulting in strain curves that were exported to a spreadsheet. Longitudinal and circumferential peak strains were defined as the greatest negative deflection, while radial strain was defined as the greatest positive value, before aortic valve closure on the strain curve. Global results were obtained by averaging the values obtained for all segments on each plane: accordingly, the LV-GLS was obtained from the mean of 18 segments acquired in the apical 4-, 2-, and 3-chamber views, while circumferential and radial strain were measured by averaging the values from all the segments of the basal, mid and apex levels. Left ventricular twist is the wringing motion of heart around its long axis. It was calculated as the net absolute difference between apical and basal rotations (LVtwist = ROTapical - ROTbasal). By widely assumed convention, apical rotation had positive values and basal, negative(20). RV-FWLS was obtained by averaging the values of the 3 right ventricular free wall segments: basal, medial, and apical. Care was taken to obtain the best visualization of the right ventricle to enable accurate delineation of its endocardial border. Irregular cardiac cycles or those containing premature ventricular beats were excluded.

The intraobserver reproducibility of strain measurements was assessed in a subsample of 30 randomly selected patients 3 months after the initial evaluation; the observer was blinded to the previous results. Interobserver variability was assessed in the same subsample by a second blinded experienced echocardiographer.

\section{Biomarkers}

Biomarker analysis was performed before EMB. For this, a $20 \mathrm{~mL}$ blood sample was collected from a peripheral vein to determine the plasma levels of cardiac troponin I and B-type natriuretic peptide (BNP).

Cardiac troponin I levels were quantified with high sensitivity 3-step sandwich immunoassay using direct chemiluminescent technology and constant amounts of 2 monoclonal antibodies. An auxiliary reagent was included to reduce nonspecific binding using an ADVIA Centaur Tnl-Ultra commercial kit (Siemens Healthcare Diagnostics, Tarrytown, New York, USA). The level of detection was $0.006 \mathrm{ng} / \mathrm{mL}$ (levels $<0.006$ are reported as $0.005 \mathrm{ng} / \mathrm{mL}$ ). The normal range of cardiac troponin I was considered $<0.04$ $\mathrm{ng} / \mathrm{mL}$. 
Plasma concentrations of BNP were determined with a 2-step sandwich immunoassay using direct chemiluminescent technology and constant amounts of 2 monoclonal antibodies using an Advia Centaur commercial kit (Siemens Healthcare, Malvern, Pennsylvania, USA). The level of detection was $2 \mathrm{pg} / \mathrm{mL}$. Levels $<2$ are reported as $1 \mathrm{pg} / \mathrm{mL}$.

\section{Statistical analysis}

Categorical variables were compared using Pearson chi-square tests, Fisher exact tests, or likelihood ratio tests. Continuous variables were compared using analysis of variance and Tukey's test (normal distribution) or Kruskal-Wallis and Dunn's tests. The results are expressed as the means with standard deviations or as the medians with interquartile ranges. Linear correlations were tested using the Spearman rank method. The sensitivity, specificity, accuracy, and positive and negative predictive values of each test for predicting ACR were calculated.

Associated risk factors of ACR $(p<0.05)$ were analyzed by were introduced in a logistic regression model with a forward stepwise approach for multivariate analysis, while receiver operating characteristic (ROC) curve analysis was conducted to determine the accuracy and an optimal cut-point value (the optimal cutpoint was assessed by jointly maximizing sensitivity and specificity).

The interobserver and intraobserver reproducibility of LV-GLS, LV-Twist and RV-FWLS were assessed using intraclass correlation coefficients and 95\% confidence intervals (Cls) in one-way random and twoway mixed models.

All analyses were performed using SPSS version 17 (SPSS Inc., Chicago, Illinois, USA). A p value $<0.05$ was considered statistically significant.

\section{Results}

Eighty-nine patients were initially included in the study. Among these patients, 29 were excluded because of the following characteristics: 2 due to cardiac arrhythmia, 4 due to left ventricular systolic dysfunction, 4 due to right ventricular systolic dysfunction, 8 due to previous ACR episodes, 4 due to a limited echocardiographic acoustic window for STE analysis, 03 due to inconclusive results of EMB, 2 due to humoral rejection and 2 due to Chagas disease recurrence. A total of 60 heart transplant patients and 60 control individuals constituted the final study population (Figure 1).

We analyzed a total of 60 EMBs of heart transplant patients. All biopsies were performed at 6 months after heart transplantation. Among these 60 EMBs, 43 (72\%) did not show significant ACR (grade 0 or 1R), while 17 (28\%) showed significant ACR (grade 2R or 3R).

The baseline characteristics of the heart transplant patients included in this study are described in Table 1. The clinical characteristics of the groups with and without significant ACR detected by EMB, as well as those of the control group individuals, are described in Table 2. 


\section{Speckle tracking echocardiography}

The variables obtained by echocardiography are shown in Table 3. There were no differences between the group with significant ACR and the group without significant ACR regarding these parameters. However, heart transplant patients had a greater septum and posterior wall thickness, larger left atrium diameter, higher $E / E^{\prime}$, and higher relative thickness and left ventricular mass index values than control individuals. In addition, heart transplant patients had lower right ventricular fractional area change, systolic velocity of the tricuspid annulus, E' velocity, A' velocity and tricuspid annular plane systolic excursion than control individuals.

The LV-GLS, LV-GCS, LV-GRS, RV-FWLS and LV-twist values are shown in Table 4. The absolute values of these variables were significantly lower in the heart transplant patients without rejection than in the control individuals, except LV-twist. In the group with significant ACR, the LV-GLS, LV-twist and RV-FWLS were significantly lower (in absolute value) than that in the group without significant ACR $(12.5 \% \pm 2.9$ vs $14.8 \% \pm 2.3, p=0.002 ; 13.9^{\circ} \pm 4.8$ vs $17.1^{\circ} \pm 3.0, p=0.048 ; 21.4 \% \pm 3.2$ vs $16.6 \% \pm 2.9, p<0.001 ;$ respectively), as shown in Figure 2. Table S1 (see the Supplementary Appendix) shows sensitivity, specificity, NPV, PPV and accuracy for each parameter related to the diagnosis of ACR degree $\geq 2 R$.

The intraclass correlation coefficient of LV-GLS was 0.92 (95\% Cl 0.85-0.96) for interobserver variability. For intraobserver variability, the intraclass correlation coefficient of the LV-GLS was $0.93(95 \% \mathrm{Cl}=0.86-$ 0.97). The intraclass correlation coefficients of the RV-FWLS were $0.90(95 \% \mathrm{Cl}=0.80-0.95)$ and 0.92 $(95 \% \mathrm{Cl}=0.84-0.96)$ for inter- and intraobserver variability, respectively. For the LV twist, the inter and intraobserver variability were $0.79(95 \% \mathrm{Cl}=0.35-0.94)$ and $0.78(0.33-0.94)$, respectively.

\section{Biomarkers}

Cardiac troponin I levels as assessed by ultrasensitive assay were significantly higher in patients with significant ACR (as determined by EMB) than in patients without significant ACR [0.19 (0.09-1.31) vs. 0.05 (0.01-0.18), $p=0.007$ ]. No significant difference in BNP levels was found between the groups, as demonstrated in Table 5.

\section{Predictors of acute cellular rejection}

A multiple logistic regression model was constructed to determine the predictive factors of ACR. In univariate analysis, the predictors of ACR were cardiac troponin I levels, RV-FWLS, LV-GLS and LV-twist. Table S2 (see the Supplementary Appendix) shows the univariate analysis using Student's t Test of the strain parameters for detection ACR. In multivariate analysis, the only significant predictor of ACR was RV-FWLS with an odds ratio of $0.57(95 \% \mathrm{Cl}=0.40-0.81 ; \mathrm{p}=0.02)$.

RV-FWLS values < 18.0\% (absolute value) presented a sensitivity of $76.5 \%$ (95\% Cl: 50.1 - 93.2), a specificity of $88.4 \%$ (95\% Cl: 74.9 - 96.1), an accuracy of $85.0 \%$ (95\% Cl: 73.4 - 92.9), a negative predictive value of $90.5 \%$ (95\% Cl: $80.1-95.8)$ and a positive predictive value of $72.2 \%(95 \% \mathrm{Cl}: 52.2-86.1)$ for detecting ACR. Figure 3 shows the ROC curve of RV-FWLS. 
Figure 4 depicts the RV-FWLS values obtained by STE and the respective anatomopathological images obtained in EMBs of heart transplant patients with and without significant ACR.

\section{Discussion}

The main finding of the present study was that RV-FWLS determined through STE is a noninvasive independent predictor of significant ACR in heart transplant patients with preserved LV and RV systolic functions. A cutoff value of $<18.0 \%$ had $85.0 \%$ accuracy for detecting significant ACR. In this population, the very strong negative predictive value of RV-FWLS may help to rule out ACR. In addition, we observed that modular values of LV-GLS and LV-twist were lower, and cardiac ultrasensitive troponin I levels was higher in transplant patients with significant ACR compared with patients without significant ACR.

In accordance with previous reports in the literature (21), we confirmed in our population that heart transplant patients had a characteristic cardiac geometric remodeling featured by greater septum and posterior wall thickness, larger left atrium diameter, and greater left ventricular mass index values than matched non transplanted controls. Heart transplant patients also showed lower values for conventional right ventricular systolic function parameters, such as fractional area change, tricuspid annulus systolic velocity and tricuspid annular plane systolic excursion. Moreover, regarding left ventricular diastolic function, heart transplant patients had lower tissue Doppler velocities and higher E/e' ratios, suggesting impaired relaxation and increased left ventricular filling pressures.

We demonstrated that heart transplant patients without rejection present unique ventricular dynamics, characterized by lower LV-GLS, LV-GCS, LV-GRS and RV-FWLS, in comparison with control individuals. We have confirmed the data recently published by Ingvarsson et al. (21), which showed that echocardiographic measurements from 124 heart transplant patients were different from reference values, unless LV-CGS. Unfortunately, the investigators did not analyze LV-Twist. In our study, we used a non-transplanted control group matched by age and sex to confirm these results. Multiple mechanisms may explain the different echocardiographic findings in heart transplant patients. The pathophysiology involves the consequences of surgical trauma, such as ischemic injury and the release of inflammatory mediators, in addition to previous pulmonary hypertension compromising right ventricular dynamics and risks associated with rejections, cardiac biopsies and immunosuppressive medications. We assume that preserved LV-twist might be responsible for maintaining cardiac function in these transplant patients without rejection.

ACR is a significant and frequent complication of heart transplantation. In the first year, it is the most common cause of mortality. Currently, EMB is the clinical gold standard in screening for graft rejection after heart transplantation and is actually the only tool for the diagnosis and classification of allograft rejection (5). Much effort has been made to improve the consistency, reliability and reproducibility of the histopathological evaluation of EMB. However, several issues make EMB assessment more difficult and less reproducible than it should be. Critical issues include the subjective and challenging pathological interpretation of EMBs and the risks associated with the procedure (22). Considering these limitations, 
noninvasive techniques to detect cardiac rejection have been evaluated, such as analysis of biomarkers (cardiac troponin I and BNP) and the use of various imaging modalities, including echocardiography, computed tomography, magnetic resonance imaging, positron emission tomography and intragraft gene expression profiling $(14,23)$.

Previous studies have shown that myocardial strain has higher sensitivity than conventional echocardiography and therefore may be an important tool to detect early subclinical cardiac dysfunction (24). Although myocardial strain imaging has been reported to have potential for the detection of graft dysfunction in the early stage $(25,26)$, its diagnostic value has not been widely recognized yet. Our study showed that the LV-GLS was lower in transplant patients with significant ACR compared with patients without significant ACR, as reported in a recent meta-analysis by Elkaryoni et al(27). When ACR occurs, myocardial deformation is impaired due to inflammatory cellular infiltration and myocardial edema and can be reflected by myocardial strain parameters. However, the diagnostic value of other strain parameters by 2D STE on ACR detection is still unknown.

Mingo-Santos et al (28). demonstrated a predictive role of STE parameters in the diagnosis of ACR (RVFWLS and LV-GLS, with threshold values of $<17 \%$ and $<15.5 \%$, respectively). That study classified biopsies into 3 groups $(0,1 R$, and $\geq 2 R)$ and was not able to detect any difference in measurements between the $1 R$ and $\geq 2 R$ groups. However, our study divided biopsy results into only two groups according to the grade of rejection: biopsies without significant rejection ( 0 and $1 R)$ and biopsies with significant rejection ( $\geq 2 \mathrm{R}$ ). This division was based upon the clinical meaning of the rejection grade, since cases with grades of 0 or $1 \mathrm{R}$ do not require immediate intervention via adjustment of immunosuppressive medications, whereas this adjustment is necessary in patients presenting with $2 \mathrm{R}$ and $3 R$ rejection. In agreement with the study from Mingo-Santos et al. (28), we confirmed the predictive value of RV-FWLS for the diagnosis of ACR, highlighting the high specificity of this parameter. RV-FWLS values $>18.0 \%$ rule out cardiac rejection with a specificity of $88.4 \%$. Our cutoff value was slightly higher than that reported by Mingo-Santos et al. (28). We speculate that it might be due to different echocardiographic equipment or even the characteristics of the studied populations.

To the best of our knowledge, this is the first study to analyze right ventricular strain using STE in patients with normal right ventricular systolic function (fractional area change $>0.34$ ) to diagnose clinically significant ACR. Extra care was given in our study to restrict patient selection to a fixed period of time (6 months post heart transplantation) to minimize the possible influence of the time since heart transplantation on right ventricular strain, as recommended by the current guidelines (29). Additionally, this fixed period of selection minimized the possible bias of pretransplantation ischemic injury, which can manifest in up to the sixth month post heart transplantation. The first six months is a period of adaptation during which many patients can still present some degree of right ventricular systolic dysfunction. The two studies that have analyzed right ventricular strain using STE to diagnose ACR did not exclude patients with fractional area change below the lower limit of normality, included individuals as early as 10 days post heart transplantation and one of them did not allocate heart rejection events according to their clinical significance $(28,30)$. 
As we show in our results, LV-twist values were significantly lower in the group with significant ACR than that in the group without significant ACR $\left(13.9^{\circ} \pm 4.79\right.$ vs $\left.17.1^{\circ} \pm 3.02, p<0.048\right)$. In parallel to our results, the unique previous study that applied 2D-STE- derived LV-twist measurement to detect rejection in heart transplanted patients have demonstrated that LV-twist decreased more in group with ACR than in group without ACR ( $9.6 \pm 2.7$ vs $12.2 \pm 2.3$ ) degrees, $p<0.0001)(31)$. We postulated that twist precede deterioration in LVEF, suggesting early myocardial involvement in cardiac rejection. With the advancement of technology that has made this technique more available and increasingly feasible, this parameter of cardiac mechanics has been increasingly studied in other pathological situations, and can be applied in this type of patient $(32,33)$.

Our study failed to find any association between diastolic markers and rejection. The results found in the literature are highly conflicting and could not be reproduced by our data. This can be explained by a limitation of diastolic dysfunction parameters due to their dependence on heart rate (which is generally elevated in transplant patients, with fusion of $E$ and $A$ waves), loading conditions and donor age (34-38).

As acute rejection promotes cardiomyocyte necrosis and results in compromised cardiac mechanics, cardiac troponin and BNP have been evaluated as potential diagnostic tools for ACR $(39,40)$. There have been controversial results on these biomarkers in the field of heart transplantation $(41,42)$. Our study used an ultrasensitive assay for cardiac troponin I that detects 10 to 100 times lower levels than standard assays. Troponin was measured before biopsy so that this procedure did not interfere with its serum levels. Troponin I levels were significantly elevated in patients with significant ACR than in those without it. However, in multivariate analysis, troponin I was not an independent predictor of ACR. Serum BNP levels were not different between groups; this finding can be explained by the suggestion of some studies that BNP remains altered in most patients for up to one year after heart transplantation $(43,44)$. Our results are in accordance with those of Bader et al. (45), who observed that BNP levels did not predict rejection at any time point after heart transplantation and suggested that BNP is not clinically useful for the detection of acute cellular rejection.

\section{Limitations}

The limitations of this study should be addressed. First, this was a single-center study with a small number of patients and a limited number of rejection episodes graded equal to or above 2R (17 out of 60 samples). Despite its extensive validation, STE is still an evolving technique, and improvements such as better tracking accuracy are still needed. Additionally, STE accuracy is highly dependent on image quality. Suboptimal resolution can produce a negative impact on the final results. Nevertheless, despite these limitations, we were able to successfully perform speckle-tracking analysis of both left and right ventricular longitudinal strain in $95 \%$ of the patients. The reproducibility of the parameters was good and was similar to that reported in other studies. Finally, our results must be independently validated in a prospective external cohort, preferably in multicenter studies, before they can be used in clinical practice.

\section{Conclusions}


Heart transplant patients have altered left ventricular dynamics compared with control individuals. STE is a valuable technique for the noninvasive detection of significant ACR in patients with heart transplant, and RV-FWLS (cutoff point $<18.0 \%$ ) is an independent predictor of ACR with good accuracy and high negative predictive value.

\section{Perspectives}

With the increasing number of heart transplantation in the world, new tools for detecting ACR is highly desirable. In our study we demonstrated that the analysis of RV-FWLS is able to predict ACR. It seems to be an accurate and noninvasive technique for analysis of heart transplant patients.

\section{Abbreviations}

ACR, Acute cellular rejection

BNP, brain natriuretic peptide

EMB, Endomyocardial biopsy

LV-GLS, left ventricular global longitudinal strain

LV-GRS, left ventricular global radial strain

LV-GCS, left ventricular global circumferential strain

RV-FWLS, right ventricular free wall longitudinal strain

STE, speckle tracking echocardiography

\section{Declarations}

\section{Ethics approval and consent to participate}

This article has been approved by the Ethics and Research Committee of the Heart Institute University of Sao Paulo

\section{Consent to publish}

All authors listed on the title page have read the manuscript, attest to the validity and legitimacy of the data and its interpretation, and agree to its submission to BMC Cardiovascular Disorders.

\section{Consent for publication}

Not applicable 
Availability of data and materials

All data generated or analysed during this study are included in this published article. If you have questions or additional information, the datasets used and/or analysed during the current study are available from the corresponding author upon reasonable request.

\section{Competing interests}

The authors declare that they have no competing interests.

\section{Authors' Contributions}

All authors contributed to the manuscript: Wilson Mathias Jr and Jeane M. Tsutsui conducted the study conceptualization, writing and review of the manuscript, Cecilia B.B.V. Cruz worked with data acquisition, analysis and writing, Ludhmila A. Hajjar and Fernando Bacal worked with clinical data acquisition of the patients, Marco L. Alves, Márcio S.M. Lima and Maria C. Abduch performed data analysis and curation, Marcelo L.C. Viera and Hsu P. Chiang performed review and editing of the manuscript, Juliana B. C. Salviano performed project administration and writing, Julia T Fukushima made the statistical analysis, Joao C.N. Sbano and Isabela B.S.C participated in the acquisition of data performed data acquisition, writing and reviewing of the manuscript. All authors have read and approved the final version of the manuscript.

\section{Acknowledgements}

Not applicable.

\section{References}

1. Luckraz H, Sharples LD, Charman SC, Tsui SS, Wallwork J, Parameshwar J, et al. Does heart transplantation confer survival benefit in all risk groups? J Heart Lung Transplant. 2005;24(9):12314.

2. Frazier $\mathrm{OH}$. Current status of cardiac transplantation and left ventricular assist devices. Tex Heart Inst J. 2010;37(3):319-21.

3. Patel JK, Kittleson M, Kobashigawa JA. Cardiac allograft rejection. Surgeon. 2011;9(3):160-7.

4. Boyle A. Current status of cardiac transplantation and mechanical circulatory support. Curr Heart Fail Rep. 2009;6(1):28-33.

5. Murphy J, Frantz R, Cooper L. Endomyocardial biopsy (123). In: Murphy J, Lloyd M, editors. Mayo Clinic Cardiology Concise Textbook. Minnesota: Rochester; 2007. p. 1481.

6. Saraiva F, Matos V, Goncalves L, Antunes M, Providencia LA. Complications of endomyocardial biopsy in heart transplant patients: a retrospective study of 2117 consecutive procedures. Transplant Proc. 2011;43(5):1908-12. 
7. Yilmaz A, Kindermann I, Kindermann M, Mahfoud F, Ukena C, Athanasiadis A, et al. Comparative evaluation of left and right ventricular endomyocardial biopsy: differences in complication rate and diagnostic performance. Circulation. 2010;122(9):900-9.

8. Holzmann M, Nicko A, Kuhl U, Noutsias M, Poller W, Hoffmann W, et al. Complication rate of right ventricular endomyocardial biopsy via the femoral approach: a retrospective and prospective study analyzing 3048 diagnostic procedures over an 11-year period. Circulation. 2008;118(17):1722-8.

9. Deckers JW, Hare JM, Baughman KL. Complications of transvenous right ventricular endomyocardial biopsy in adult patients with cardiomyopathy: a seven-year survey of 546 consecutive diagnostic procedures in a tertiary referral center. J Am Coll Cardiol. 1992;19(1):43-7.

10. Zakliczynski M, Nozynski J, Konecka-Mrowka D, Pyka L, Trybunia D, Swierad M, et al. Quilty effect correlates with biopsy-proven acute cellular rejection but does not predict transplanted heart coronary artery vasculopathy. J Heart Lung Transplant. 2009;28(3):255-9.

11. Pardo-Mindan FJ, Lozano MD. "Quilty effect" in heart transplantation: is it related to acute rejection? J Heart Lung Transplant. 1991;10(6):937-41.

12. Forbes RD, Rowan RA, Billingham ME. Endocardial infiltrates in human heart transplants: a serial biopsy analysis comparing four immunosuppression protocols. Human pathology. 1990;21(8):8505.

13. Wu AH, Feng YJ, Moore R, Apple FS, McPherson PH, Buechler KF, et al. Characterization of cardiac troponin subunit release into serum after acute myocardial infarction and comparison of assays for troponin T and I. American association for clinical chemistry subcommittee on cTnl standardization. Clin Chem. 1998;44(6 Pt 1):1198-208.

14. Holweg CT, Potena L, Luikart H, Yu T, Berry GJ, Cooke JP, et al. Identification and classification of acute cardiac rejection by intragraft transcriptional profiling. Circulation. 2011;123(20):2236-43.

15. Fitzsimons S, Evans J, Parameshwar J, Pettit SJ. Utility of troponin assays for exclusion of acute cellular rejection after heart transplantation: A systematic review. J Heart Lung Transplant. 2018;37(5):631-8.

16. Garrido IP, Pascual-Figal DA, Nicolas F, Gonzalez-Carrillo MJ, Manzano-Fernandez S, Sanchez-Mas J, et al. Usefulness of serial monitoring of B-type natriuretic peptide for the detection of acute rejection after heart transplantation. The American journal of cardiology. 2009;103(8):1149-53.

17. Stewart S, Winters GL, Fishbein MC, Tazelaar HD, Kobashigawa J, Abrams J, et al. Revision of the 1990 working formulation for the standardization of nomenclature in the diagnosis of heart rejection. J Heart Lung Transplant. 2005;24(11):1710-20.

18. Lang RM, Badano LP, Mor-Avi V, Afilalo J, Armstrong A, Ernande L, et al. Recommendations for cardiac chamber quantification by echocardiography in adults: an update from the American Society of Echocardiography and the European Association of Cardiovascular Imaging. Eur Heart $\mathrm{J}$ Cardiovasc Imaging. 2015;16(3):233-70.

19. Devereux RB, Alonso DR, Lutas EM, Gottlieb GJ, Campo E, Sachs I, et al. Echocardiographic assessment of left ventricular hypertrophy: comparison to necropsy findings. The American journal 
of cardiology. 1986;57(6):450-8.

20. Mor-Avi V, Lang RM, Badano LP, Belohlavek M, Cardim NM, Derumeaux G, et al. Current and evolving echocardiographic techniques for the quantitative evaluation of cardiac mechanics: ASE/EAE consensus statement on methodology and indications endorsed by the Japanese Society of Echocardiography. Eur J Echocardiogr. 2011;12(3):167-205.

21. Ingvarsson A, Evaldsson AW, Waktare J, Nilsson J, Smith GJ, Stagmo M, et al. Normal reference ranges for transthoracic echocardiography following heart transplantation. Journal of the American Society of Echocardiography: official publication of the American Society of Echocardiography. 2018;31(3):349-60.

22. Strecker T, Rosch J, Weyand M, Agaimy A. Endomyocardial biopsy for monitoring heart transplant patients: 11-years-experience at a german heart center. Int J Clin Exp Pathol. 2013;6(1):55-65.

23. Wu YL, Ye Q, Ho C. Cellular and functional imaging of cardiac transplant rejection. Curr Cardiovasc Imaging Rep. 2011;4(1):50-62.

24. Geyer H, Caracciolo G, Abe H, Wilansky S, Carerj S, Gentile F, et al. Assessment of myocardial mechanics using speckle tracking echocardiography: fundamentals and clinical applications. J Am Soc Echocardiogr. 2010;23(4):351-69. quiz 453-5.

25. Tseng AS, Gorsi US, Barros-Gomes S, Miller FA, Pellikka PA, Clavell AL, et al. Use of speckle-tracking echocardiography-derived strain and systolic strain rate measurements to predict rejection in transplant hearts with preserved ejection fraction. BMC Cardiovasc Disord. 2018;18(1):241.

26. Sera F, Kato TS, Farr M, Russo C, Jin Z, Marboe CC, et al. Left ventricular longitudinal strain by speckle-tracking echocardiography is associated with treatment-requiring cardiac allograft rejection. J Card Fail. 2014;20(5):359-64.

27. Elkaryoni A, Altibi AM, Khan MS, Okasha O, Ellakany K, Hassan A, et al. Global longitudinal strain assessment of the left ventricle by speckle tracking echocardiography detects acute cellular rejection in orthotopic heart transplant recipients: A systematic review and meta-analysis. Echocardiography. 2020;37(2):302-9.

28. Mingo-Santos S, Monivas-Palomero V, Garcia-Lunar I, Mitroi CD, Goirigolzarri-Artaza J, Rivero B, et al. Usefulness of Two-Dimensional Strain Parameters to Diagnose Acute Rejection after Heart Transplantation. J Am Soc Echocardiogr. 2015;28(10):1149-56.

29. Badano LP, Miglioranza MH, Edvardsen T, Colafranceschi AS, Muraru D, Bacal F, et al. European Association of Cardiovascular Imaging/Cardiovascular Imaging Department of the Brazilian Society of Cardiology recommendations for the use of cardiac imaging to assess and follow patients after heart transplantation. Eur Heart J Cardiovasc Imaging. 2015;16(9):919-48.

30. Antonczyk K, Niklewski T, Antonczyk R, Zakliczynski M, Zembala M, Kukulski T. Speckle-Tracking Echocardiography for Monitoring Acute Rejection in Transplanted Heart. Transplant Proc. 2018;50(7):2090-4.

31. Sato T, Kato TS, Komamura K, Hashimoto S, Shishido T, Mano A, et al. Utility of left ventricular systolic torsion derived from 2-dimensional speckle-tracking echocardiography in monitoring acute 
cellular rejection in heart transplant recipients. J Heart Lung Transplant. 2011;30(5):536-43.

32. Zhang Y, Li SY, Xie JJ, Wu Y. Twist/untwist parameters are promising evaluators of myocardial mechanic changes in heart failure patients with preserved ejection fraction. Clin Cardiol. 2020.

33. Jia FW, Hsu J, Liu XH, Feng XJ, Pang HY, Lin X, et al. Usefulness of Myocardial Strain and Twist for Early Detection of Myocardial Dysfunction in Patients With Autoimmune Diseases. The American journal of cardiology. 2020;125(3):475-81.

34. Mena C, Wencker D, Krumholz HM, McNamara RL. Detection of heart transplant rejection in adults by echocardiographic diastolic indices: a systematic review of the literature. J Am Soc Echocardiogr. 2006;19(10):1295-300.

35. Dandel M, Hummel M, Muller J, Wellnhofer E, Meyer R, Solowjowa N, et al. Reliability of tissue Doppler wall motion monitoring after heart transplantation for replacement of invasive routine screenings by optimally timed cardiac biopsies and catheterizations. Circulation. 2001;104(12 Suppl 1):1184-91.

36. Sun JP, Abdalla IA, Asher CR, Greenberg NL, Popovic ZB, Taylor DO, et al. Non-invasive evaluation of orthotopic heart transplant rejection by echocardiography. J Heart Lung Transplant. 2005;24(2):1605.

37. Sade LE, Sezgin A, Ulucam M, Taymaz S, Simsek V, Tayfun E, et al. Evaluation of the potential role of echocardiography in the detection of allograft rejection in heart transplant recipients. Transplant Proc. 2006;38(2):636-8.

38. Stengel SM, Allemann Y, Zimmerli M, Lipp E, Kucher N, Mohacsi P, et al. Doppler tissue imaging for assessing left ventricular diastolic dysfunction in heart transplant rejection. Heart. 2001;86(4):4327.

39. Zimmermann R, Baki S, Dengler TJ, Ring GH, Remppis A, Lange R, et al. Troponin T release after heart transplantation. Br Heart J. 1993;69(5):395-8.

40. Dengler TJ, Zimmermann R, Braun K, Muller-Bardorff M, Zehelein J, Sack FU, et al. Elevated serum concentrations of cardiac troponin $T$ in acute allograft rejection after human heart transplantation. $J$ Am Coll Cardiol. 1998;32(2):405-12.

41. Fitzsimons S, Evans J, Parameshwar J, Pettit SJ. Utility of troponin assays for exclusion of acute cellular rejection after heart transplantation: a systematic review. J Heart Lung Transplant. 2018;37(5):631-8.

42. Talha S, Charloux A, Piquard F, Geny B. Brain natriuretic peptide and right heart dysfunction after heart transplantation. Clin Transplant. 2017;31(6):http://dx.doi:10.1111/ctr.12969.

43. Hervas I, Almenar L, Perez-Pastor JL, Chirivella M, Osa A, Martinez-Dolz L, et al. Radioimmunometric assay of B-type natriuretic peptide (BNP) in heart transplantation: correlation between BNP determinations and biopsy grading of rejection. Nucl Med Commun. 2003;24(8):925-31.

44. Gardner RS, Chong KS, Murday AJ, Morton JJ, McDonagh TA. N-terminal brain natriuretic peptide is predictive of death after cardiac transplantation. Heart. 2006;92(1):121-3. 
45. Bader FM, Rogers RK, Kfoury AG, Gilbert EM, Horne BD, Stehlik J, et al. Time-dependent changes in Btype natriuretic peptide after heart transplantation: correlation with allograft rejection and function. Congest Heart Fail. 2009;15(2):63-7.

\section{Tables}

Table 1. Baseline characteristics of the transplant patients. 


\begin{tabular}{|c|c|}
\hline \multirow[t]{2}{*}{ Characteristic } & Heart transplant patients \\
\hline & $n=60$ \\
\hline Male sex & $34(56.7 \%)$ \\
\hline Age (years) & $42.1 \pm 11.5$ \\
\hline Weight (kg) & $61.3 \pm 13.4$ \\
\hline Height (cm) & $162.5 \pm 10.3$ \\
\hline Body surface area $\left(\mathrm{m}^{2}\right)$ & $1.7 \pm 0.2$ \\
\hline \multicolumn{2}{|l|}{ Primary disease } \\
\hline Chagasic cardiomyopathy & $22(36.7 \%)$ \\
\hline Idiopathic dilated cardiomyopathy & $21(35.0 \%)$ \\
\hline Ischemic cardiomyopathy & $7(11.7 \%)$ \\
\hline Valvular cardiomyopathy & $5(8.3 \%)$ \\
\hline Hypertensive cardiomyopathy & $4(6.7 \%)$ \\
\hline CTRCD & $1(1.6 \%)$ \\
\hline Hypertension & $12(20.0 \%)$ \\
\hline Diabetes & $12(20.0 \%)$ \\
\hline Smoking & $17(28.3 \%)$ \\
\hline Time since transplantation (months) & 6 \\
\hline \multicolumn{2}{|l|}{ Medication } \\
\hline Corticosteroid & $63(95.5 \%)$ \\
\hline Mycophenolate mofetil & $62(93.9 \%)$ \\
\hline Tacrolimus & $45(68.2 \%)$ \\
\hline Cyclosporine & $21(31.8 \%)$ \\
\hline Sirolimus & $4(6.1 \%)$ \\
\hline Azathioprine & $4(6.1 \%)$ \\
\hline Statin & $61(92.4 \%)$ \\
\hline CCB & $56(84.5 \%)$ \\
\hline Betablocker & $11(16.7 \%)$ \\
\hline ACEI / ARB & $11(16.7 \%)$ \\
\hline
\end{tabular}

Page 18/25 
The values are presented as the mean (standard deviation) or number (percentage).

CTRCD = Cancer therapeutic-related cardiac dysfunction;

ACEI = angiotensin converting enzyme inhibitor; $\mathrm{ARB}$ = aldosterone receptor blocker

$\mathrm{CCB}=$ calcium channel blocker

Table 2. Group characteristics by rejection status.

\begin{tabular}{|lllll|}
\hline Variable & Control group & Group without & Group with & p value \\
\cline { 2 - 5 } & $(\mathrm{n}=60)$ & significant ACR $(\mathrm{n}=43)$ & significant ACR $(\mathrm{n}=17)$ & \\
\hline Age (years) & $43 \pm 9$ & $44 \pm 11$ & $48 \pm 13$ & $0.20^{\mathrm{c}}$ \\
\hline Male sex & $34(56.7 \%)$ & $24(55.8 \%)$ & $10(58.8 \%)$ & $0.139^{\mathrm{b}}$ \\
\hline Weight $(\mathrm{kg})$ & $72(63-80)$ & $70(58-81)$ & $71(61-87)$ & $0.684^{\mathrm{a}}$ \\
\hline Height $(\mathrm{cm})$ & $1.69 \pm 0.09$ & $1.62 \pm 0.09^{\#}$ & $1.59 \pm 0.13^{\#}$ & $<0.001^{\mathrm{c}}$ \\
\hline BMI $\left(\mathrm{kg} / \mathrm{m}^{2}\right)$ & $24.7(22.9-27.1)$ & $26.0(22.7-31.6)$ & $26.1(24.0-34.7)$ & $0.152^{\mathrm{a}}$ \\
\hline SBP $(\mathrm{mmHg})$ & $123 \pm 13$ & $126 \pm 19$ & $122 \pm 13$ & $0.672^{\mathrm{c}}$ \\
\hline DBP $(\mathrm{mmHg})$ & $76 \pm 10$ & $76 \pm 10$ & $72 \pm 8$ & $0.463^{\mathrm{c}}$ \\
\hline PAP $(\mathrm{mmHg})$ & - & $30 \pm 10$ & $34 \pm 11$ & $0.465^{\mathrm{d}}$ \\
\hline Hypertension & $0(0 \%)$ & $7(16.3 \%)$ & $5(29.4 \%)$ & $<0.001^{\mathrm{b}}$ \\
\hline Diabetes mellitus & $0(0 \%)$ & $7(16.3 \%)$ & $5(29.4 \%)$ & $<0.001^{\mathrm{b}}$ \\
\hline Smoking & $0(0 \%)$ & $14(32.6 \%)$ & $3(17.6 \%)$ & $<0.001^{\mathrm{b}}$ \\
\hline Cr (mg/dL) & $0.80(0.5-1.0)$ & $1.19(0.9-1.34)$ & $1.1(0.95-1.4)$ & $0.930^{\text {a }}$ \\
\hline BSA (m $\left.{ }^{2}\right)$ & $1.60(1.55-1.68)$ & $1.78(1.58-1.93)^{\#}$ & $1.75(1.60-2.03)^{\#}$ & $<0.001^{\text {a }}$ \\
\hline
\end{tabular}

The values are presented as the mean (standard deviation), median (interquartile range) or number (percentage). $\mathrm{BMI}=$ body mass index; $\mathrm{Cr}=$ creatinine; $\mathrm{BSA}=$ body surface area; SBP, Systolic blood pressure; DBP, diastolic blood pressure; PAP, pulmonary artery pressure.

${ }^{a}$ Kruskal-Wallis test; ${ }^{b}$ Pearson chi-square; ${ }^{c}$ ANOVA

$\# p<0.05$ (vs. the control group) 
Table 3. Echocardiographic parameters and their relationships with significant acute cellular rejection in univariate analysis.

\begin{tabular}{|c|c|c|c|c|}
\hline \multirow[t]{2}{*}{ Variable } & Control & \multirow{2}{*}{$\begin{array}{l}\text { Group } \\
\text { without ACR }\end{array}$} & \multirow{2}{*}{$\begin{array}{l}\text { Group } \\
\text { with ACR }\end{array}$} & \multirow[t]{2}{*}{$p$ value } \\
\hline & group & & & \\
\hline Left atrium $(\mathrm{mm})$ & $34(31-36)$ & $41(38-46) \#$ & 43 (35-49)\# & $<0.001^{\mathrm{a}}$ \\
\hline LA Volume $\left(\mathrm{mm}^{3} / \mathrm{cm}^{2}\right)$ & $25.02 \pm 2.97$ & $33.31 \pm 4.96 \#$ & $33.78 \pm 3.06 \#$ & $<0.001^{b}$ \\
\hline Septum (mm) & $8(8-9)$ & $11(10-13) \#$ & $12(10-13) \#$ & $<0.001^{\mathrm{a}}$ \\
\hline Posterior wall (mm) & $8(8-9)$ & $11(10-11) \#$ & $11(9-13) \#$ & $<0.001^{\mathrm{a}}$ \\
\hline Relative thickness & $\begin{array}{l}0.38(0.35- \\
0.41)\end{array}$ & 0.49 (0.44-0.53)\# & $\begin{array}{l}0.47(0.40- \\
0.55) \#\end{array}$ & $<0.001^{\mathrm{a}}$ \\
\hline LVMI $\left(\mathrm{g} / \mathrm{m}^{2}\right)$ & $71(65-83)$ & $102(86-124) \#$ & 109 (80-141)\# & $<0.001^{\mathrm{a}}$ \\
\hline LVEF (\%) & $65(62-68)$ & $64(62-67)$ & $65(63-67)$ & $0.739^{a}$ \\
\hline E velocity (m/s) & $\begin{array}{l}0.84(0.69- \\
0.93)\end{array}$ & $\begin{array}{l}0.65(0.60-0.76) \\
\#\end{array}$ & $\begin{array}{l}0.66(0.54-0.84) \\
\#\end{array}$ & $<0.001^{\mathrm{a}}$ \\
\hline DT (ms) & $\begin{array}{l}189(168- \\
208)\end{array}$ & 187 (144-214) & 190 (153-207) & $0.787^{a}$ \\
\hline A velocity (m/s) & $\begin{array}{l}0.50(0.42- \\
0.58)\end{array}$ & $\begin{array}{l}0.41(0.32-0.50) \\
\#\end{array}$ & $\begin{array}{l}0.42(0.38-0.68) \\
\#\end{array}$ & $0.001^{\mathrm{a}}$ \\
\hline $\mathrm{E} / \mathrm{A}$ & $\begin{array}{l}1.60(1.38- \\
1.93)\end{array}$ & $1.64(1.29-2.00)$ & $1.46(1.29-1.75)$ & $0.543^{a}$ \\
\hline $\mathrm{S}^{\prime}$ velocity $(\mathrm{m} / \mathrm{s})$ & $\begin{array}{l}0.08(0.07- \\
0.09)\end{array}$ & 0.07 (0.06-0.08)\# & $0.07(0.05-0.08)$ & $0.010^{\mathrm{a}}$ \\
\hline$E^{\prime}$ velocity (m/s) & $\begin{array}{l}0.11(0.09- \\
0.12)\end{array}$ & $0.06(0.05-0.08) \#$ & $\begin{array}{l}0.07(0.05- \\
0.07) \#\end{array}$ & $<0.001^{\mathrm{a}}$ \\
\hline$A^{\prime}$ velocity $(\mathrm{m} / \mathrm{s})$ & $\begin{array}{l}0.08(0.07- \\
0.10)\end{array}$ & $0.06(0.05-0.08) \#$ & $\begin{array}{l}0.08(0.06- \\
0.09) \#\end{array}$ & $<0.001^{\mathrm{a}}$ \\
\hline E/E' & $\begin{array}{l}7.92(6.63- \\
8.78)\end{array}$ & $\begin{array}{l}10.14(7.99- \\
12.96) \#\end{array}$ & $\begin{array}{l}10(7.93- \\
14.21) \#\end{array}$ & $<0.001^{\mathrm{a}}$ \\
\hline FAC (\%) & $43(40-48)$ & 39 (36-45) \# & 39 (37-42) \# & $0.001^{\mathrm{a}}$ \\
\hline TAPSE (mm) & $24(20-26)$ & $17(15-18) \#$ & 16 (15-17)\# & $<0.001^{\mathrm{a}}$ \\
\hline $\begin{array}{l}\mathrm{S}^{\prime} \text { velocity }(\mathrm{m} / \mathrm{s}) \text { of tricuspid } \\
\text { annulus }\end{array}$ & $13(12-15)$ & $12(11-14)$ & $11(11-12) \#$ & $<0.001^{\mathrm{a}}$ \\
\hline
\end{tabular}


The values are presented as the mean (standard deviation) or median (interquartile range).

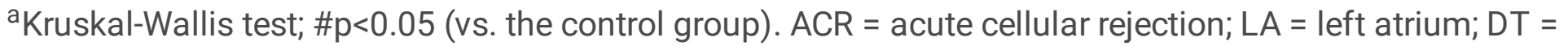
deceleration time; $L V E F=$ left ventricular ejection fraction; $F A C=$ fractional area change; lat = lateral; LVMI $=$ left ventricular mass index; TAPSE = tricuspid annular plane systolic excursion.

Table 4. LV-twist, LV-GLS, LV-GCS, LV-GRS and RV-FWLS values and their relationships with cardiac rejection in univariate analysis.

\begin{tabular}{|c|c|c|c|c|c|c|c|}
\hline \multirow[t]{3}{*}{ Variable } & Control & Without & With & \multirow[t]{3}{*}{$P^{+}$} & \multirow[t]{3}{*}{$P^{*}$} & \multirow[t]{3}{*}{$P^{*}$} & \multirow[t]{2}{*}{ Overall } \\
\hline & group & ACR & ACR & & & & \\
\hline & $(n=60)$ & $(n=43)$ & $(n=17)$ & & & & $P$ value ${ }^{\mathcal{S}}$ \\
\hline LV-twist $\left({ }^{\circ}\right)$ & $18.8 \pm 4.63$ & $17.1 \pm 3.02$ & $13.9 \pm 4.79$ & 0.402 & 0.002 & 0.048 & 0.001 \\
\hline LV-GLS (\%) AV & $21.2 \pm 2.1$ & $14.8 \pm 2.3$ & $12.5 \pm 2.9$ & $<0.001$ & $<0.001$ & 0.002 & $<0.001$ \\
\hline LV-GCS (\%) ${ }^{A V}$ & $21.3 \pm 3.0$ & $16.2 \pm 4.3$ & $15.2 \pm 2.8$ & $<0.001$ & $<0.001$ & 0.574 & $<0.001$ \\
\hline LV-GRS (\%) & $41.2 \pm 12.9$ & $28.6 \pm 9.9$ & $28.3 \pm 8.8$ & $<0.000$ & $<0.001$ & 0.996 & $<0.001$ \\
\hline RV-FWLS (\%) ${ }^{A V}$ & $25.0 \pm 3.3$ & $21.4 \pm 3.2$ & $16.6 \pm 2.9$ & $<0.000$ & $<0.001$ & $<0.001$ & $<0.001$ \\
\hline \multicolumn{8}{|c|}{$\mathrm{AV}=$ absolute value } \\
\hline \multicolumn{8}{|c|}{$\mathrm{ACR}=$ acute cellular rejection } \\
\hline
\end{tabular}

Data are expressed as mean \pm SD.

† Control group vs group without significant ACR.

¥Control group vs group with significant ACR.

${ }^{*}$ Group without ACR vs group with significant ACR

- Analysis of variance global P value between groups.

Table 5. Biomarkers and their relationships with acute cellular rejection in univariate analysis. 


\begin{tabular}{|llll|}
\hline Variable & $\begin{array}{l}\text { Group } \\
\text { without ACR }\end{array}$ & $\begin{array}{l}\text { Group } \\
\text { with ACR }\end{array}$ & p value \\
\hline Troponin I (ng/mL) & $0.05(0.01$ to 0.18$)$ & $0.19(0.09$ to 1.31$)$ & $0.007^{*}$ \\
\hline BNP $(\mathrm{pg} / \mathrm{mL})$ & $248(90$ to 528$)$ & $264.5(160$ to 976$)$ & $0.435^{*}$ \\
\hline
\end{tabular}

The values are presented as the median (interquartile range). *Mann-Whitney

$\mathrm{BNP}=$ brain natriuretic peptide; $\mathrm{ACR}=$ acute cellular rejection.

\section{Figures}

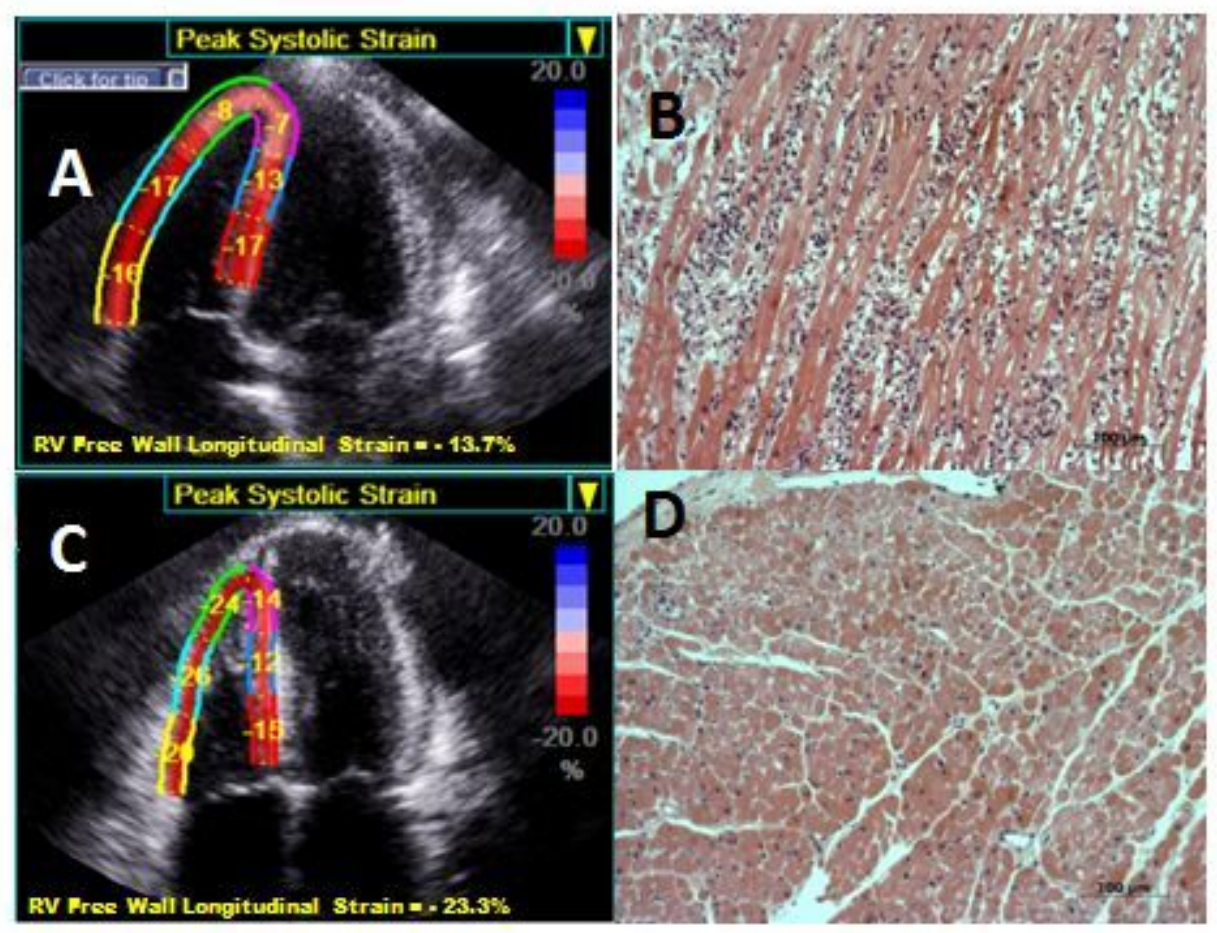

\section{Figure 1}

Right ventricle free wall longitudinal strain (RV-FWLS) and endomyocardial biopsy in patients with heart transplants. The images were obtained in an apical four-chamber view. A) RV-FWLS in a heart transplant patient demonstrating a low value $(-13.7 \%)$ and $B$ ) the corresponding endomyocardial biopsy showing acute cellular rejection (ACR). C) RV-FWLS in a heart transplant patient demonstrating a higher value ($23.3 \%$ ) and $D$ ) the corresponding endomyocardial biopsy without signs of ACR. To calculate RV-FWLS, we averaged the longitudinal strain values for the 3 right ventricular free wall segments (basal, medial, and apical). The interventricular septum segments were excluded. 


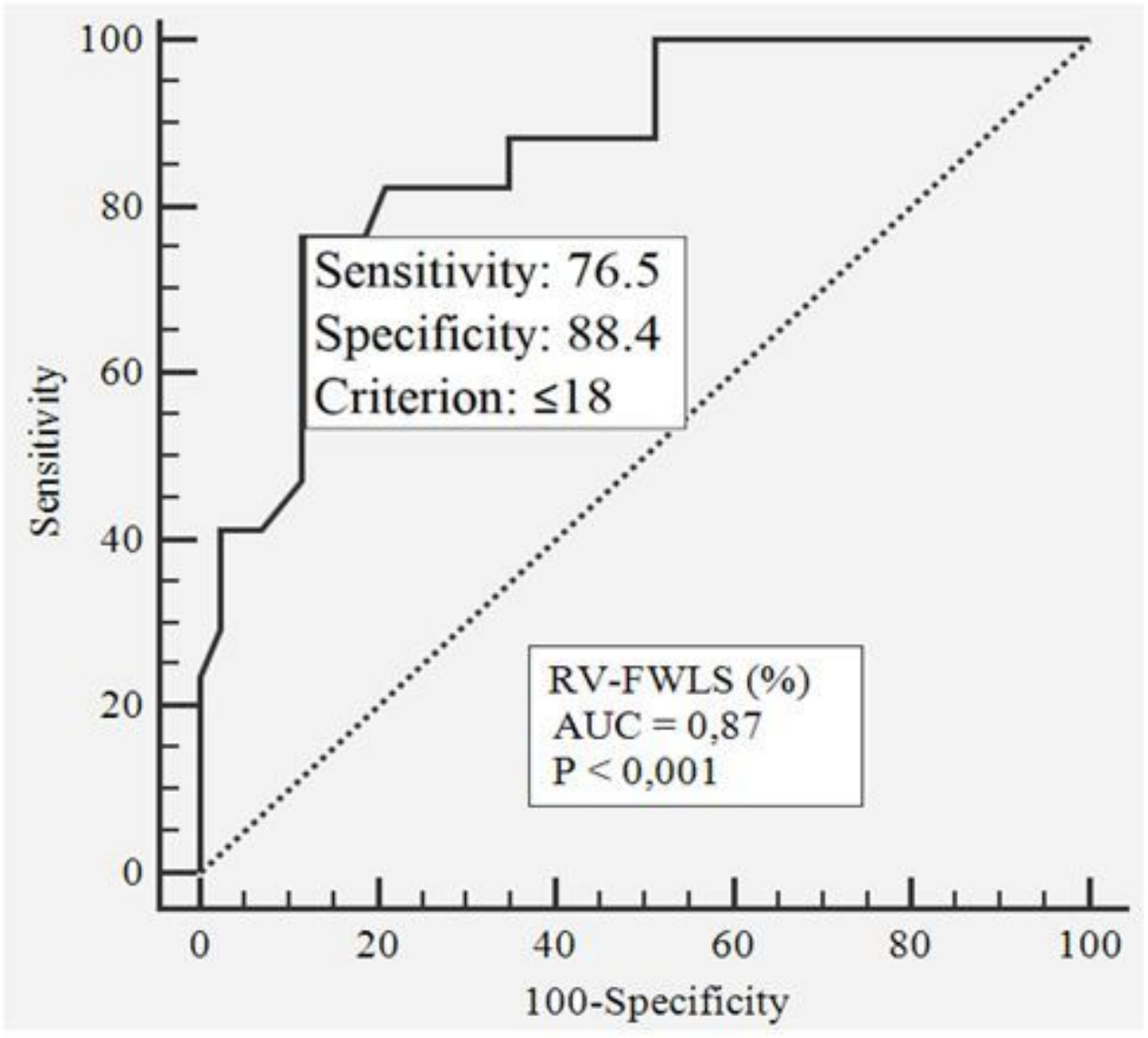

Figure 2

Receiver operating characteristic curve demonstrating the right ventricular free wall longitudinal strain values obtained by speckle tracking echocardiography for the diagnosis of acute cellular rejection in heart transplant patients. AUC = area under curve. 


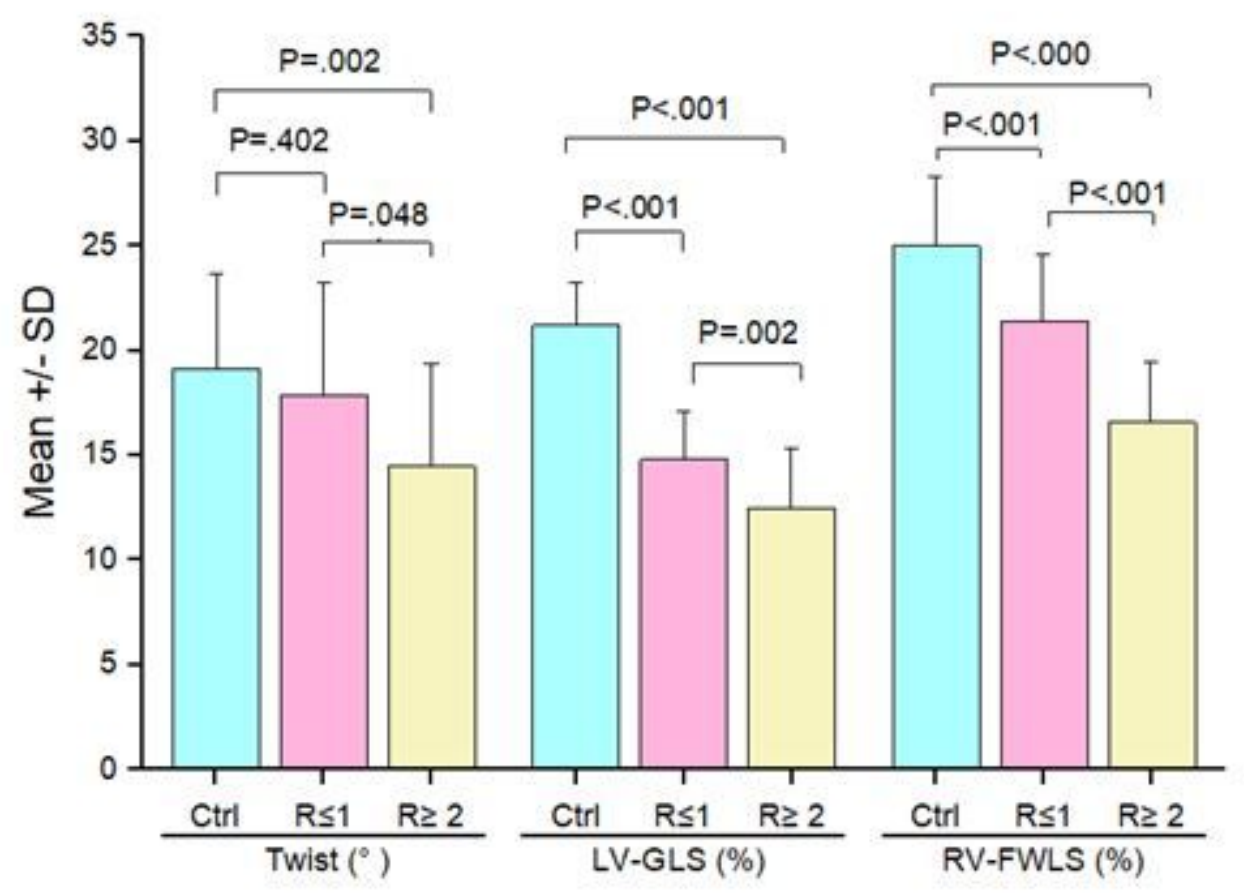

\section{Figure 3}

Twist, left ventricular global longitudinal strain and right ventricular free wall longitudinal strain in the control group and in two groups of heart transplant patients, those with and those without significant acute cellular rejection ( $R \geq 2$ vs. $R \leq 1$ ) ANOVA: F-test. *Significative to $5 \%$ (Tukey test for all pairwise comparisons); Ctrl = Control (Cardiac untransplanted patients); $\mathrm{R} \leq 1$, Heart transplant patients without rejection ; $R \geq 2$, Heart transplant patients with rejection $\geq 2 R$. SEM: Mean with error bars representing standard error of the mean; LV-GLS: Left Ventricular Global Longitudinal Strain; RV-FWLS: Right Ventricle Free Wall Longitudinal Strain. 


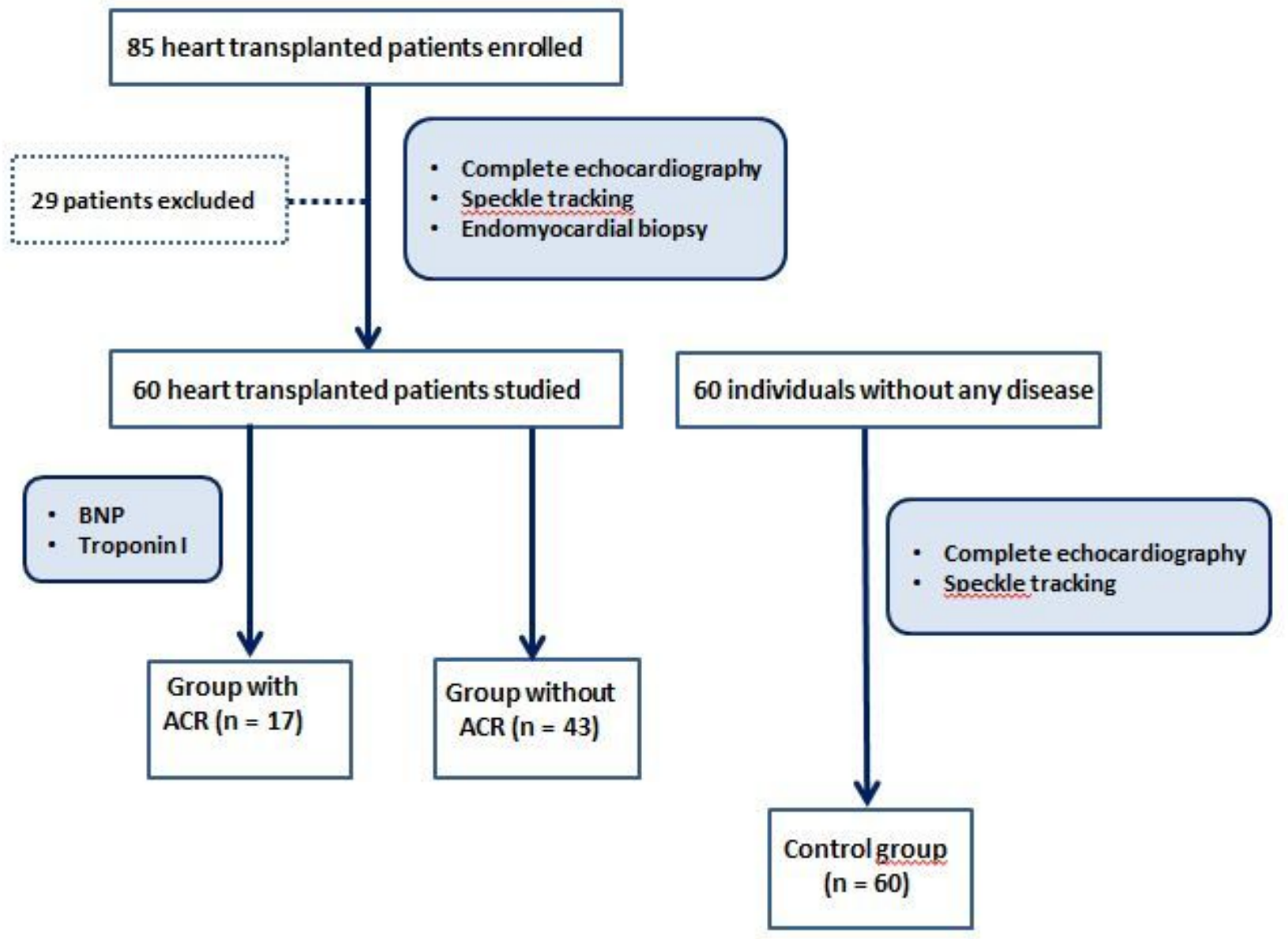

Figure 4

Study flow diagram. EMB = endomyocardial biopsy; $A C R=$ acute cellular rejection.

\section{Supplementary Files}

This is a list of supplementary files associated with this preprint. Click to download.

- SupplementaryAppendixACR.docx 